\title{
Deposition of ITO thin films onto PMMA substrates for waveguide based biosensing devices
}

\author{
S. Azevedo ${ }^{1, a}$, L. Diéguez ${ }^{2,3}$, P. Carvalho ${ }^{1}$, J.O. Carneiro ${ }^{1}$, V. Teixeira ${ }^{1, b}$, \\ E. Martínez ${ }^{2,4}$ and J. Samitier ${ }^{2,3,4}$ \\ ${ }^{1}$ Centre of Physics, University of Minho, Azurém Campus, 4800-058 Guimarães, Portugal \\ ${ }^{2}$ Nanobioengineering group, Institute for Bioengineering of Catalonia (IBEC), Baldiri Reixac 10-12, \\ Barcelona, Spain \\ ${ }^{3}$ Department of Electronics, University of Barcelona, Barcelona, Spain \\ ${ }^{4}$ Networking Research Center on Bioengineering, Biomaterials and Nanomedicine (CIBER-BBN), \\ Barcelona, Spain \\ acsofiaazevedo@fisica.uminho.pt, ${ }^{b}$ vasco@fisica.uminho.pt (corresponding authors)
}

Keywords: Waveguide biosensing devices; ITO thin films; PMMA sheets

\begin{abstract}
Biosensors' research filed has clearly been changing towards the production of multifunctional and innovative design concepts to address the needs related with sensitivity and selectivity of the devices. More recently, waveguide biosensors, that do not require any label procedure to detect biomolecules adsorbed on its surface, have been pointed out as one of the most promising technologies for the production of biosensing devices with enhanced performance. Moreover the combination of optical and electrochemical measurements through the integration of transparent and conducting oxides in the multilayer structures can greatly enhance the biosensors' sensitivity. Furthermore, the integration of polymeric substrates may bring powerful advantages in comparison with silicon based ones. The biosensors will have a lower production costs being possible to disposable them after use ("one use sensor chip"). This research work represents a preliminary study about the influence of substrate temperature on the overall properties of ITO thin films deposited by DC magnetron sputtering onto 0,5 mm thick PMMA sheets.
\end{abstract}

\section{Introduction}

A biosensor is capable of perform a quantitative or semi-quantitative analysis through the integration of a biological recognition element with an appropriate signal transducer element. Normally biosensors are classified in terms of the transducer used (e.g. electrochemical, optical, piezoelectric and thermal, among others)[1]. Optical biosensors, that use an optical sensor as a signal transducer, are considered a good choice since they allow non destructive measurements. Additionally, some label-free detection mechanisms measure the change induced in refractive index by molecular interactions, related to surface density or sample concentration, instead of total mass [2].

Waveguide based optical sensors are of increasing interest for biosensor applications due to their high sensitivity to minor refractive index changes near to sensor surface in an aqueous environment [3]. Thin film optical waveguides employed in guided-wave devices of integrated optics consist of three different materials, one for the substrate, one for the film and the third for the cover. Classical waveguide structures for sensor applications consist of a high refractive index thin film $\left(1.8<\mathrm{n}_{\mathrm{F}}<\right.$ 2.2) deposited on a glass or polymer substrate with a lower refractive index $\left(1.45<\mathrm{n}_{\mathrm{S}}<1.55\right)$. Generally,for biosensor applications, the medium that covers the waveguide containing the analyte to be probed is aqueous $\left(\mathrm{n}_{\mathrm{C}}=1.33\right)$. So, $\mathrm{n}_{\mathrm{F}}>\mathrm{n}_{\mathrm{S}}>\mathrm{n}_{\mathrm{C}}$. [4]. 
To allow the construction of complex device structures, with dimensions ranging from hundreds of microns down to a few tens of nanometers or less, materials cannot be expensive and also should be simple to machine. When required, materials have to be compatible with fluidic applications and provide rigid and smooth surfaces [5]. Common materials that satisfy the above requirements are based on silicon (pure silicon, glass or quartz) and carbon (in the form of polymers and plastics).

The last group has become increasingly used especially in biological sciences, development of polymer based apparatus, such as lab-on-a-chip devices and biochips. Their unique properties, such as strength, transparency, deformability allied to simple manufacturing techniques and low cost, made them an alternative to silicon based materials [6]. Apart from these characteristics polymer structures are cheaper to produce than silicon-based fabrication technologies, thereby normally dispensing the need of high-energy or multi-step fabrication techniques [6].

Poly(methyl methacrylate) - PMMA - is an amorphous, transparent and colorless thermoplastic polymer with a glass transition temperature $(T \mathrm{~g})$ of about $105^{\circ} \mathrm{C}$ [7,8]. It has good abrasion and UV resistance and excellent optical clarity but poor temperature, fatigue and solvent resistances [8]. It is hard and stiff, with low thermal-expansion and pressure-shrinkage coefficients $\left(\sim 5 \times 10^{-5}\right.$ per ${ }^{\circ} \mathrm{C}$ and $\sim 5 \times 10-11 \mathrm{~Pa}^{-1}$, respectively), making it a perfect candidate for imprinting techniques. However, this is counterbalanced by its optical properties (colorlessness, transparency, and UV resistance), which, together with its excellent optical clarity, make it ideal for use in the production of biomedical devices [7]. Although its outstanding advantages for biosensors production PMMA has a drawback when optical biosensors based on refractive index changes are under development. The polymer has a low refractive index (1.492), not meeting the necessary requirements [7]. The problem can be solved by the deposition of a transparent thin film with higher refractive index such as indium tin oxide on the PMMA surface.

As a result of their wide range of technical applications, oxide thin films that are both transparent and electrically conducting have been extensively studied. For label free biosensing, a combination of lightmode spectroscopy, voltammetry and/or potentiometric electrochemical techniques offer more precision in the detection and identification of the biomolecules and biocells. Transparent conductive oxides (TCO's) are an interesting alternative to the metallic transparent thin films, because of their high transparency and good conductivity. The most extensively used TCO is Indium Tin Oxide (ITO) [8]. The attractiveness of ITO is related to the low sheet resistance (Rs), high optical transmittance in the visible region of the electromagnetic spectrum and its high refractive index. However this properties are very hard to attain because are strongly dependent of the growth conditions. Moreover, it is well known that high quality ITO films are easily obtained when substrate are heated up to $250-350^{\circ} \mathrm{C}$ [9]. ITO thin films have been produced by almost all physical and chemical methods [10-12]. Reproducible ITO films can be produced by reactive evaporation (Yamaguchi, et al. 447 (2004) 115), reactive DC or RF sputtering [13], sol-gel process [14] and chemical vapor deposition (CVD) [15].

It is important to refer that, for practical application and industrial production, is required an improvement of the method to grow ITO thin films at room temperature for dissimilar applications (such as biosensing devices) $[16,17]$.

\section{Materials and Methods}

The ITO thin films were deposited on the surface of $0.5 \mathrm{~mm}$ thick PMMA sheets and conventional microscope slides by using DC magnetron sputtering of a $75 \mathrm{~mm}$ oxide target $\left(\mathrm{In}_{2} \mathrm{O}_{3}: \mathrm{SnO}_{2}=90: 10 \mathrm{wt} . \%\right)$. Before deposition, the sputtering chamber was pumped down to $3.5 \times 10^{-5}$ mbar using a combinatory system of a rotary pump (Alcatel ANNECY Ty-2033, PS $-33 \mathrm{~m}^{3} / \mathrm{h}$ ) and a diffusion pump (Edwards Vapor Pump EO4, PS - 6001/s), which work together to maintain high vacuum. More details about the vacuum system used in this work can be found in reference [18]. Prior to each deposition, the ITO ceramic target was pre-sputtered during $5 \mathrm{~min}$. 
Only the substrate temperature of sputtering parameters was varied in order to investigate its effect on film properties. Thin films were deposited on glass and PMMA substrates first without any intentional heating of the substrate and then with different substrate temperatures of 80,90 and $100^{\circ} \mathrm{C}$ (lower than the transition glass temperature of PMMA $-\mathrm{T}_{\mathrm{g}}=105^{\circ} \mathrm{C}$ to avoid the degradation of the polymer properties). In Table 1 is presented a summary of the deposition parameters used. The substrate temperature was varied in order to understand its influence in the overall properties of the ITO thin films.

Table 1 - Sputtering deposition parameters.

\begin{tabular}{|c|c|}
\hline Parameter & Value \\
\hline Base Pressure (mbar) & $3.5 \times 10^{-5}$ \\
\hline Working Pressure (mbar) & $2.7 \times 10^{-3}$ \\
\hline Deposition time $(\mathrm{min})$ & 5 \\
\hline Oxygen flow $(\mathrm{sccm})$ & 0.8 \\
\hline Argon flow $(\mathrm{sccm})$ & 7.5 \\
\hline Target Current $(\mathrm{mA})$ & 200 \\
\hline Target/substrate distance $(\mathrm{mm})$ & 80 \\
\hline Deposition Temperature $\left({ }^{\circ} \mathrm{C}\right)$ & $\mathrm{RT}, 80,90,100$ \\
\hline
\end{tabular}

$\mathrm{X}$-ray diffraction (XRD) analyses were carried out to in order to evaluate the crystalline structure of the ITO thin films deposited onto PMMA substrates. XRD $2 \theta$ scans were recorded by using a Cuk $\mathrm{Cu}_{\alpha}$ radiation source in a Philips $P W 1710$ X-ray Diffractometer. The surface morphology and roughness of the produced thin films was observed in an atomic force microscopy (AFM) from Digital Instruments controlled by Nanoscope III software. The thickness of the ITO thin films was measured through the observation of the ITO cross-section by using scanning electron microscopy (SEM) in the Leica Cambridge S360 instrument. The electrical properties (resistivity, carrier concentration and Hall Mobility) of ITO thin films were measured by van der Pauw method. The optical transmittance spectra were recorded in a Shimadzu UV-310PC Scanning Spectrophotometer.

\section{Results and discussion}

The deposition rate of the ITO thin films is $0.4 \mathrm{~nm} / \mathrm{s}$ which corresponds to a thickness of about $120 \mathrm{~nm}$ which was corroborated by SEM analysis to the samples cross section. In Figure 1 are presented the diffraction patterns of two ITO thin films deposited on the surface of PMMA sheets with any intentional heating (hereafter designed by RT) and $90^{\circ} \mathrm{C}$. 


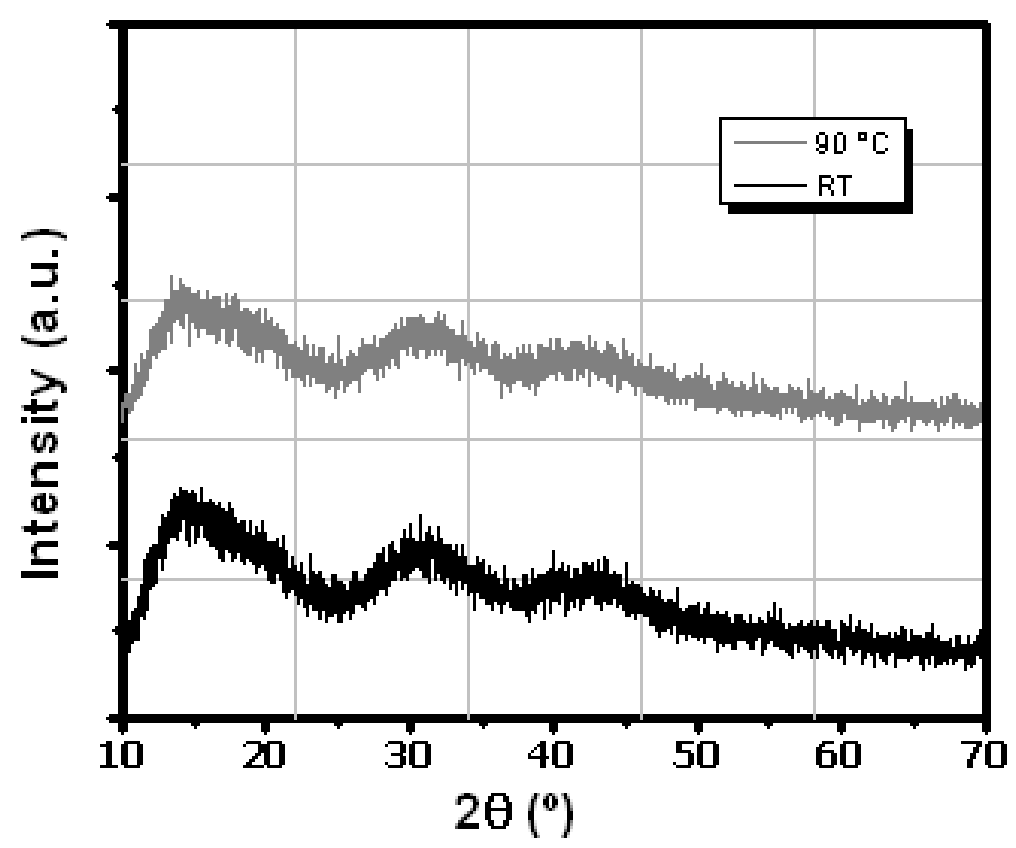

Fig. 1 - Diffractograms of samples deposited onto PMMA sheets at room temperature and with $90^{\circ} \mathrm{C}$ of substrate temperature.

The ITO films deposited are amorphous; they do not have any sharp peak in their X-Ray diffractogram. Only a weak broad background from the PMMA substrate can be observed. These results also confirm that this range of temperature does not affect the crystallinity of the thin films. The attained results are in agreement with other work carried out in the same deposition chamber [16]. Also, as mentioned by Mohamed, S. H. and coworkers [19]: when lower substrate temperatures are used the polymer substrate tailors the ITO structure towards amorphous state.

In Figure 2 b) is presented the AFM results of ITO thin films deposited on PMMA substrates. To have a comparison reference, in figure 2a) it is also shown the surface morphology of a PMMA bare substrate.
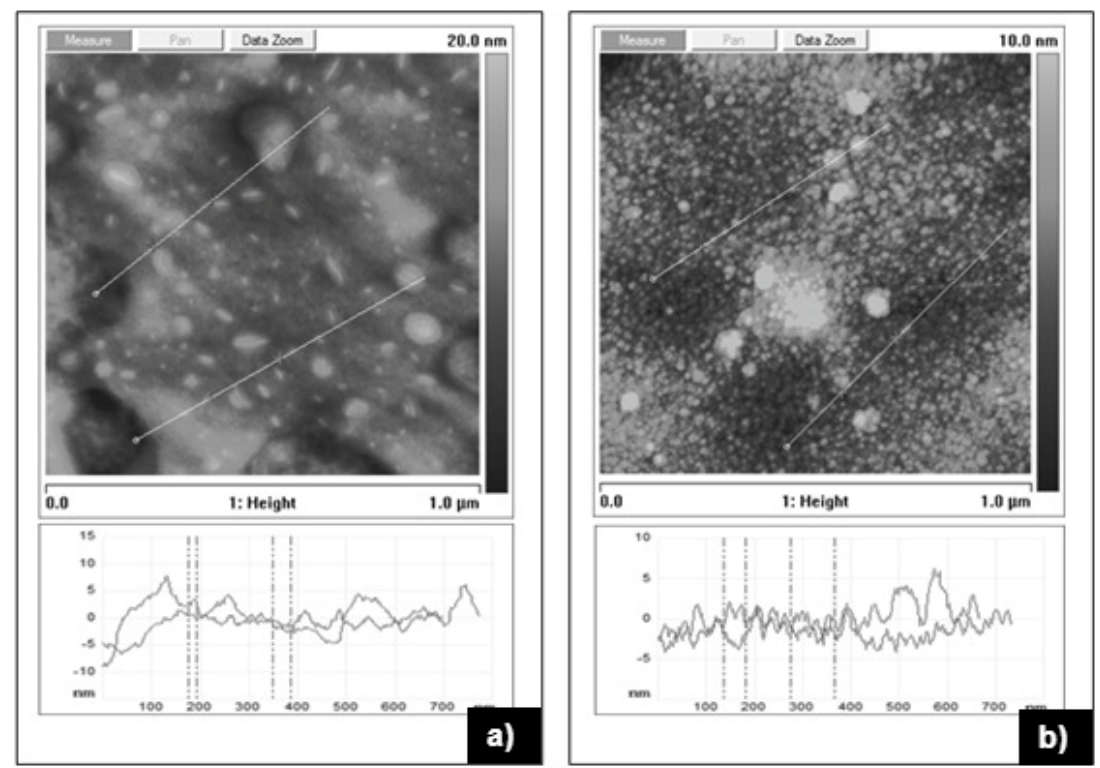

Fig. 2 - AFM images of a) PMMA substrate and b) ITO deposited on PMMA at RT. 
Figure 3 a) and b) display the AFM images of ITO thin films deposited on glass substrates without any intentionaly heating the substrates and at $100{ }^{\circ} \mathrm{C}$.
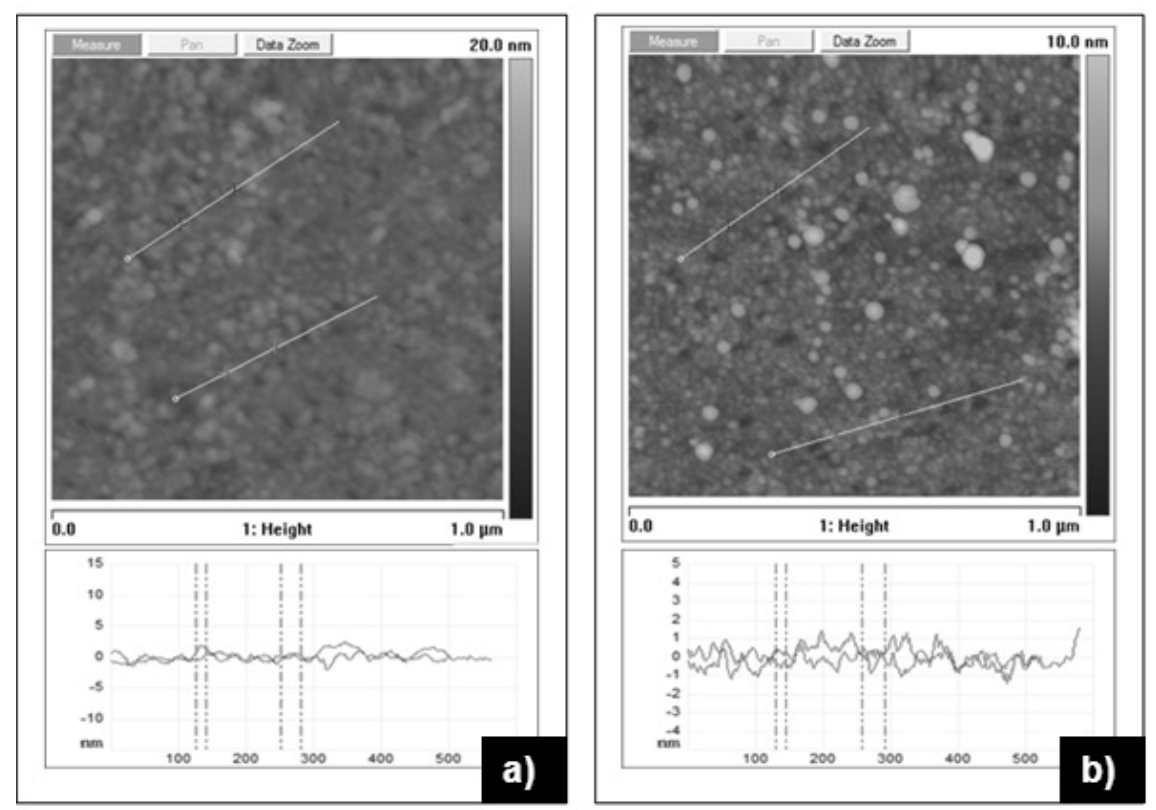

Fig. 3 - AFM images of ITO deposited on glass substrate at a) RT and b) $100^{\circ} \mathrm{C}$.

From the analysis of the previous results it is possible to conclude that the surface morphology of the ITO is clearly dependent of the substrate used. Additionally, as shows Figure 2a), the PMMA substrates have poor surface quality: there are dissimilar holes and scratches that may interfere in the optical properties of the to-be-developed devices. ITO thin films deposited on the surface of glass substrates are smoother than the ones deposited on to surface of the PMMA substrates.

The influence of substrate temperature on the ITO thin films deposited on PMMA and glass substrates was determined by measuring the average roughness $(\mathrm{Ra})$ of the produced samples. The results are presented in the graph of Figure 4.

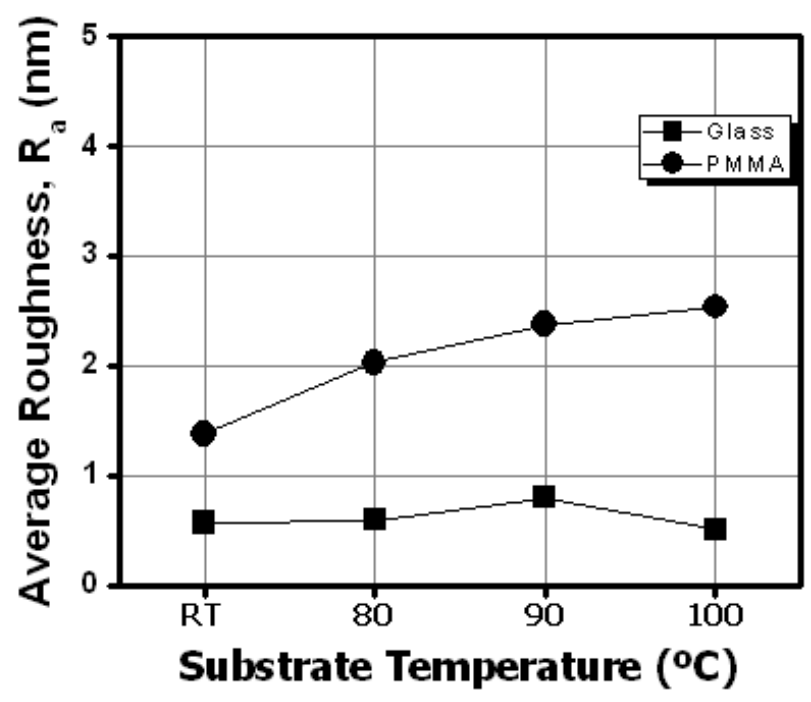

Fig. 4 - Graphic showing the influence of substrate temperature on the average roughness $\left(R_{a}\right)$ of ITO thin films deposited on glass and PMMA substrates. 
The roughness of ITO deposited on PMMA substrates increase with substrate temperature, whereas for glass substrate the roughness does not change with substrate heating. At room temperature the ITO thin films deposited onto PMMA sheets have an average roughness of about $1.3 \mathrm{~nm}$ whereas when deposited on glass substrate the measured average roughness was $0.6 \mathrm{~nm}$. This is about $50 \%$ of the value obtained for coated PMMA substrates.

Although an effort has been made to deposit the ITO thin films bellow the glass transition temperature of PMMA $\left(\mathrm{Tg}=105^{\circ} \mathrm{C}\right)$, when the substrates are heated up to $80{ }^{\circ} \mathrm{C}, 90^{\circ} \mathrm{C}$ and $100{ }^{\circ} \mathrm{C}$, the highly energetic collisions of the sputtered species with the substrate can further increase the substrate temperature. In this case, if the $\mathrm{Tg}$ is exceeded some structural changes may happens to the polymer and thus leading to an increase in the thin films roughness.

The optical properties if the ITO thin films deposited on both substrates were characterized by measuring their transmittance spectra. The obtained results can be seen in the graphics of figures $5 \mathrm{a}$ ) ad b). For both cases, the transmittance spectra of the substrates are also presented to have a comparison reference.

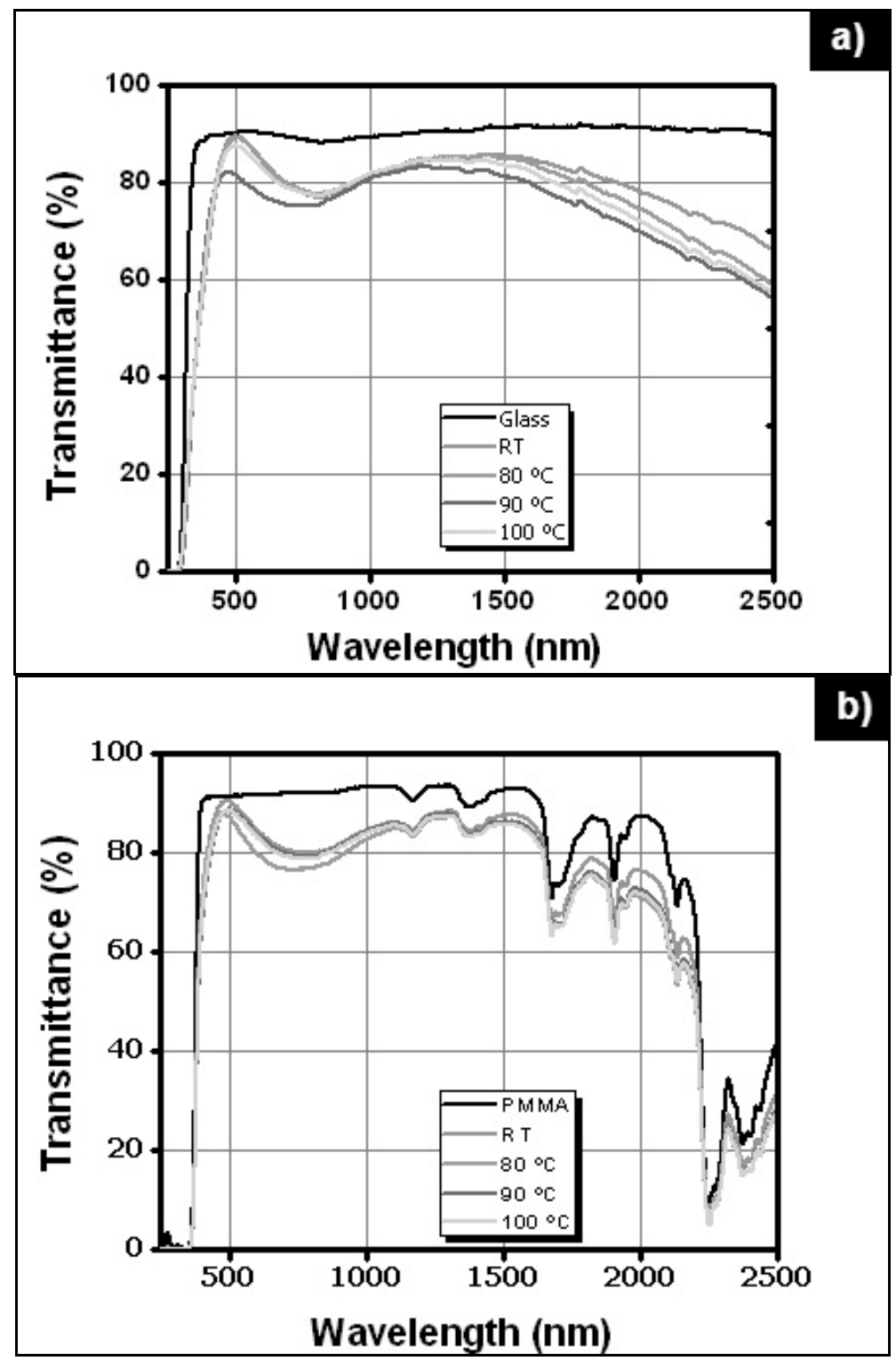

Figure 5 - Transmittance Spectrum of ITO thin films deposited on a) glass and b) PMMA substrates with different substrate temperature. 
The deposited ITO thin films on PMMA and glass substrates were transparent having high transmittance $(\mathrm{T} \%)$ in the visible region of the electromagnetic spectrum. In table 2 are presented the results of the average transmittance in the visible region and also at $632.8 \mathrm{~nm}$. This wavelength was chosen since it is commonly used a He-Ne laser for waveguide biosensors devices.

Table 2 - Average transmittance in the visible wavelength $\left(T_{m}\right)$ and transmittance at $632.8 \mathrm{~nm}$ (T@632.8nm) for ITO samples deposited at different substrate temperatures on glass and PMMA substrates.

\begin{tabular}{|l|l|l|l|l|}
\hline & $\begin{array}{l}\mathbf{T}_{\mathbf{m}}(\boldsymbol{\%}) \\
\text { Glass }\end{array}$ & $\begin{array}{l}\text { T @632.8nm } \\
\text { Glass }\end{array}$ & $\begin{array}{l}\mathbf{T}_{\mathbf{m}}(\boldsymbol{\%}) \\
\text { PMMA }\end{array}$ & $\begin{array}{l}\text { T @632.8nm } \\
\text { PMMA }\end{array}$ \\
\hline RT & 82.76 & 82.56 & 83.34 & 83.06 \\
\hline $\mathbf{8 0}^{\mathbf{}} \mathbf{C}$ & 82.65 & 82.82 & 80.30 & 78.61 \\
\hline $\mathbf{9 0}^{\mathbf{C}} \mathbf{C}$ & 77.83 & 76.55 & 82.26 & 82.46 \\
\hline $\mathbf{1 0 0}^{\circ} \mathbf{C}$ & 81.72 & 81.29 & 81.99 & 81.86 \\
\hline
\end{tabular}

From Table 2 it is possible to conclude that samples deposited on glass and PMMA substrates have high optical transmittance in the visible region (about 80\%). Regarding the application aimed for these films, probably the best sample will be the one deposited at room temperature since concerning PMMA substrates it has the highest optical transmittance at $632.8 \mathrm{~nm}$ (He-Ne laser wavelength) and the lowest roughness.

The electrical properties of ITO thin films deposited on glass substrates were characterized by Hall Effect measurements using van der Pauw method with which was possible to obtain their sheet resistance, electrical resistivity, carrier concentration and carrier mobility. In Figure 6 are shown the electrical resistivity results a function of substrate temperature.

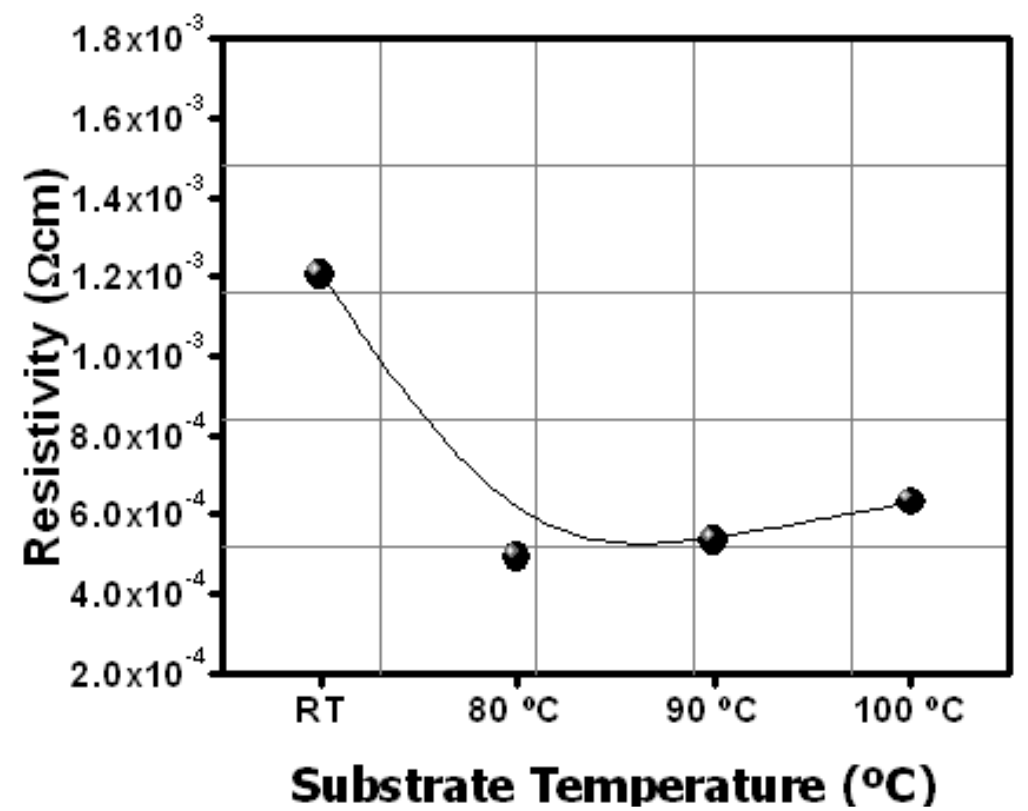

Fig. 6 - Electrical resistivity as a function of substrate temperature of ITO thin films deposited on glass substrates.

From the results presented in the last graph it is possible to see that samples deposited with higher substrate temperature have lower electrical resistivity (about $5 \times 10^{-4} \Omega \mathrm{cm}$ ) in comparison with sample deposited at room temperature $\left(1.21 \times 10^{-3} \Omega \mathrm{cm}\right)$. The sample deposited at $80^{\circ} \mathrm{C}$ has the higher conductivity. Carrier concentration and Hall mobility are important criteria to describe the electrical performance of ITO thin films. These results are presented in Figure 7. 


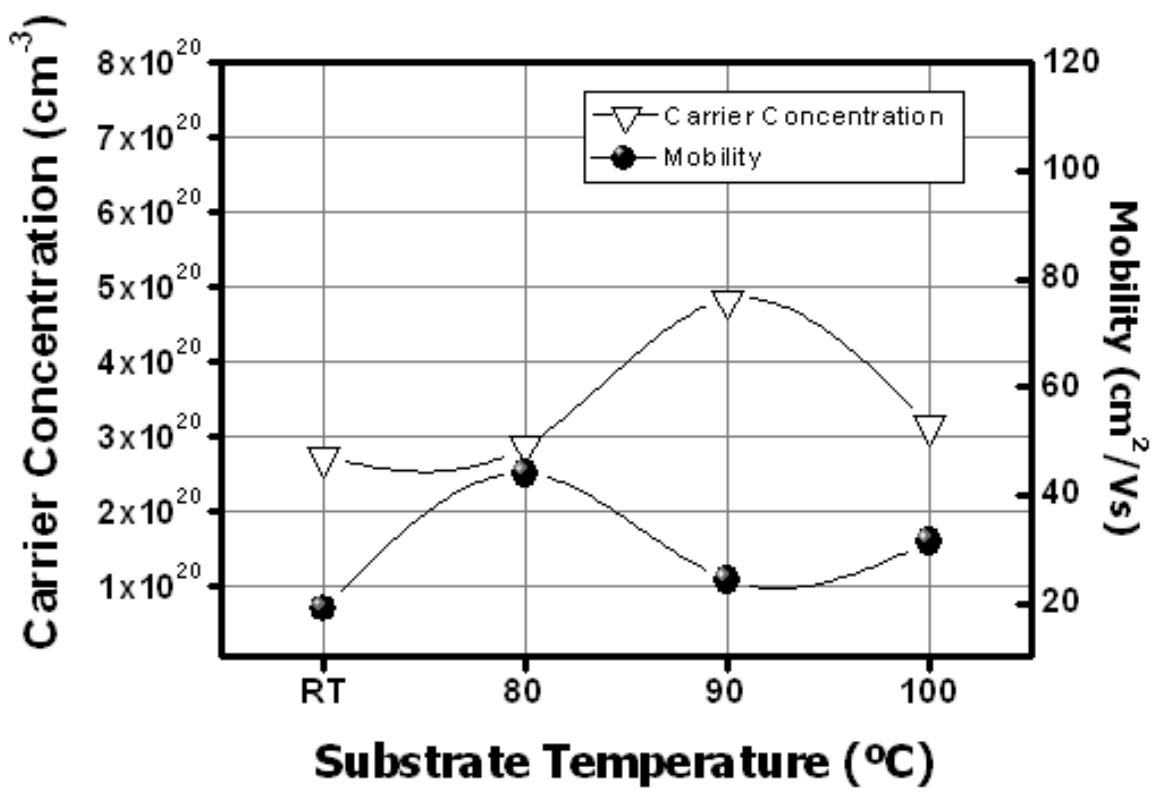

Fig.7 - Carrier concentration and Hall mobility as a function of substrate temperature.

The high electrical resistance of sample deposited at room temperature is due to low carrier concentration and low mobility. The species reaching substrate, at room temperature, have low mobility, so that will remain in the place where they impinge (not reaching its equilibrium position). This can promote the scattering centers that lead to an increase of electrical resistivity by decreasing the mobility of charge carrier [20].

\section{Conclusions}

Indium tin oxide thin films were deposited on PMMA and glass substrates by using DC magnetron sputtering, with the purpose to define the best deposition parameters to produce a transparent and conducting thin film.

Based on the application aimed, the best results were attained when no intentional heating was applied to the substrates. Although the conductivity is lower in comparison to the obtained values for samples deposited at higher temperatures, the optical transmittance of the ITO thin film deposited at room temperature is higher and the surface roughness if the lowest measured. Changing other parameters such as the oxygen flux, target voltage and/or distance between the target and the substrate may lead to an improvement in the electrical properties.

\section{Acknowledgements}

The authors acknowledge FCT for Sofia Azevedo Scholarship under the scope of the project NANO/NMED-SD/0076/2007-"NanoMeDiag- Nanobioanalytical platforms for improved medical diagnosis of infections caused by pathogen microorganisms" (project leader (Spain): J. Samitier, Portuguese leader: V. Teixeira). 


\section{References}

[1] J.P.Chambers, B.P. Arulanandam, L.L. Matta, A. Weis, J.J. Valdes, Biosensor recognition elements. Current Issues of Molecular Biology 10 (2008), 1-12.

[2] X. Fan, I M. White, S.I. Shopova, H. Zhu, J. D. Suter and Y. Sun, Sensitive optical biosensors for unlabeled targets: A review, Analytical Chimica Acta 620 (2008) 8-26.

[3] A. Densmore, D.-X. Xu, S. Janz, P. Waldron, J. Lapointe, T. Mischki, G. Lopinski, A. Delâge, J.H. Scmid and P. Cheben, Sensitive Label-Free Biomolecular Detection Using Thin Silicon Waveguides, Advances in Optical Technologies (2008)

[4] R. Horváth , L. R Lindvold and N. B Larsen, Fabrication of all-polymer freestanding waveguides, Journal of Micromechanics and microengineering, 13 (2003) 419-424.

[5] C. A. Mills, E. Martinez, A. Errachid, G. Gomila, A. Samsó, and J. Samitier, Small Scale Structures. The Fabrication of Polymeric Nanostructures for Biomedical Applications using Pattern Replication Techniques, Contributions to science 3(1): 47-56 (2005).

[6] C.A. Mills, J. G. Fernandez, A.Errachid, J. Samitier, The use of high glass temperature polymers in the production of transparent, structured surfaces using nanoimprint lithography Microelectronic Engineering. 85 (2008) 1897-1901.

[8] Ch. Sujatha, G. Mohan Rao, S. Uthanna, Characteristics of indium tin oxide films deposited by bias magnetron sputtering, Materials Science and Engineering, B 94 (2002) 106-110.

[9] V. Teixeira, H. N. Cui, L.J. Meng, E. Fortunato, and R. Martins. Amorphous ITO thin films prepared by DC sputtering for electrochromic applications Thin Solid Films , 420-421 (2002) 70-75.

[10]J. S. Kim, , M. Granstrom, and R.H. Friend. Journal of Applied Physics, 84 (1998) 6869.

[11] E. Shanthi, A. Bannerjee, and V. Dutta. Annealing characteristics of tin oxide films prepared by spray pyrolysis Thin Solid Films, 71 (1980) 237.

[12] J.C. Jin, and I. Hamberg. Applied Physics Letters , 57 (1987) 149.

[13] N. Danson, I. Safi, G.W. Hall, and R.P. Howson. Techniques for the sputtering of optimum indium-tin oxide films on to room-temperature substrates Surface Coating Technology, 99 (1998) 147.

[14] M. Alam, and D. Cameron. Optical and electrical properties of transparent conductive ITO thin films deposited by sol-gel process Thin Solid Films, 377 (2000) 455.

[15] K. Maki, N. Komiya, and A. Suzuki. Fabrication of thin films of ITO by aerosol CVD Thin Solid Films, 445 (2003) 224.

[16] Cui, H-N., V. Teixeira, and A. Monteiro. Microstructure study of indium tin oxide thin films by optical methods Vacuum, 67 (2002) 589-594.

[17] Lee, J., H. Junga, D. Limb, and K. Yangb. Effects of bias voltage on the properties of ITO films prepared on polymer substrates Thin Solid Films, 480-481 (2005) 157- 161.

[18] J. O. Carneiro, V. Teixeira, A. João, A. Magalhães, C. Tavares Study of Nd-doping effect and mechanical cracking on photoreactivity of $\mathrm{TiO}_{2}$ thin films Vacuum. 82 (2008) 1475-1481.

[19] S.H. Mohamed, F.M. El-Hossary, G.A. Gamal, and M.M. Kahlid. Acta Physica Polonia A, 115 (2009) 704-708.

[20] Monteiro, A. Revestimentos multicamada PVD com comportamento electrocrómico, Tese de Mestrado. Braga: Universidade do Minho, 2004. 
Journal of Nano Research Vol. 17

10.4028/www.scientific.net/JNanoR.17

Deposition of ITO Thin Films onto PMMA Substrates for Waveguide Based Biosensing Devices

10.4028/www.scientific.net/JNanoR.17.75 